\title{
Entre patrimoine et développement durable, quel avenir pour les églises paroissiales?
}

Étude sur le territoire de la région urbaine Lyon Saint-Étienne

Between heritage and sustainable development: what future for churches?

Field study of the Lyon Saint-Étienne urban area

\section{Mélanie Meynier-Philip}

\section{OpenEdition}

Journals

Édition électronique

URL : http://journals.openedition.org/developpementdurable/12058

DOI : $10.4000 /$ developpementdurable. 12058

ISSN : $1772-9971$

\section{Éditeur}

Association DD\&T

\section{Référence électronique}

Mélanie Meynier-Philip, « Entre patrimoine et développement durable, quel avenir pour les églises paroissiales? ", Développement durable et territoires [En ligne], Vol. 9, n¹ | Mars 2018, mis en ligne le 30 mars 2018, consulté le 30 avril 2019. URL : http://journals.openedition.org/

developpementdurable/12058 ; DOI : 10.4000/developpementdurable.12058

Ce document a été généré automatiquement le 30 avril 2019.

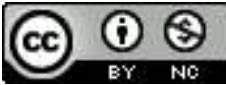

Développement Durable et Territoires est mis à disposition selon les termes de la licence Creative Commons Attribution - Pas d'Utilisation Commerciale 4.0 International. 


\title{
Entre patrimoine et développement durable, quel avenir pour les églises paroissiales?
}

\author{
Étude sur le territoire de la région urbaine Lyon Saint-Étienne \\ Between heritage and sustainable development: what future for churches? \\ Field study of the Lyon Saint-Étienne urban area
}

Mélanie Meynier-Philip

1 Cet article porte sur la reconversion des églises dans un contexte de sécularisation des sociétés occidentales. En effet, la baisse de la pratique des cultes historiques, soit la religion catholique pour la France (Hervieu-Léger, 2003), a engendré un phénomène de désaffectation des églises. Face à ce constat, l'avenir de ces édifices est envisagé à travers leur valeur d'usage et leur valeur affective. La première est étudiée au regard de leur potentiel de reconversion et de leur prédisposition à se renouveler, phénomène historiquement observé (Cieren, 2014), lorsque le culte s'arrête. La seconde, observée à travers des mobilisations citoyennes à chaque annonce de fermeture, permet d'envisager l'acceptabilité sociale de ces reconversions. La pratique de la reconversion, autrement dit la flexibilité architecturale (Kronenburg, 2007), rejoint la notion de durabilité et les préoccupations sociétales où les enjeux environnementaux sont de plus en plus affirmés.

2 Les études en sciences humaines et sociales portant sur les périodes modernes et contemporaines ont fortement investi ce phénomène de sécularisation (Hervieu-Léger, 1999 ; Desmoulins-Hémery et Palouzié, 2006 ; Faltraueur et al., 2012 ; Fornerod, 2013 ; Coomans et Sauvé, 2014). Pourtant, rares sont celles qui se penchent sur les conséquences de la désaffectation récente des lieux de culte, due à une baisse de la pratique religieuse ${ }^{1}$ et observée à travers des édifices sous-utilisés, voire non utilisés (Fornerod, 2013). Encore moins nombreuses sont celles qui abordent la question des moyens pour assurer leur reconversion leur reconversion architecturale et fonctionnelle.

3 En 2005, pour la première fois Luc Noppen et Lucie K. Morisset traitent cette problématique lors d'un colloque international intitulé «Quel avenir pour quelles 
églises ?² ». Au Québec, la recherche de solutions d'avenir pour les églises désaffectées a une longueur d'avance du fait de son histoire ${ }^{3}$. La France est aujourd'hui à son tour confrontée à la problématique de conservation/démolition, car de nombreuses églises ferment ou sont déjà fermées, voire démolies. L'Observatoire du patrimoine religieux estime que près de 5 à $10 \%$ des églises catholiques pourraient être vendues, détruites ou abandonnées d'ici à 2030 soit entre 5000 et 10000 édifices $^{4}$.

Ce phénomène connaît une accélération considérable et mérite d'être appréhendé dans une vision globale prenant en compte l'ensemble des problématiques territoriales rurales, comme de renouvellement urbain et de développement durable.

Quand une église perd son usage premier, la question de sa conservation se pose. Lors de la nationalisation des biens de l'Église à la Révolution, l'histoire a largement montré l'adaptabilité de ces édifices, à travers de nombreuses utilisations spontanées en écurie, halle de grains ou manège d'équitation (Cieren, 2014). Aujourd'hui, la sacralité qui entoure ces édifices semble occulter les possibilités de transformation. Même si une étude ${ }^{5}$ affirme que $71 \%$ des catholiques français sont favorables à la transformation en «bâtiments civils» des églises vacantes, les représentations sociales autour de ces bâtiments demeurent conservatrices. La dimension symbolique inhérente au principe de réutilisation des églises est puissante, et a des conséquences sur l'attitude que nous avons face à cette situation. De fait, l'inaction est favorisée et de nombreuses églises se dégradent au point où la démolition semble être l'unique solution économiquement viable.

6 Au-delà de l'aspect mémoriel, qui fédère unanimement les opinions (Duboscq et Moulinier, 1987; Maurey, 2015), pourquoi garder les églises, quel sens peut-on leur donner aujourd'hui et quels sont les enjeux de cette patrimonialisation d'un point de vue durable? Quels sont les impacts environnementaux lorsqu'une démolition est inévitable? La question de la gestion des déchets n'est pas propre à ce corpus, en revanche la particularité des matériaux qui constituent une église, principalement des matériaux de gros œuvre nous le verrons, est à envisager pour développer des stratégies de réemploi (Huygen, 2008) plutôt que de recyclage, alliant mémoire et ressource existante, comme cela existe pour d'autres édifices et d'autres types de matière.

7 Après une définition de la notion de développement durable, une analyse des liens entre cette définition et la notion de patrimoine qui a subi un élargissement depuis les dernières décennies, nous mettrons en évidence comment le patrimoine, incarné ici par les églises, s'inscrit dans une logique de durabilité. Nous verrons comment cette dynamique durable est possible grâce au processus de patrimonialisation qui entraîne la conservation et la valorisation du bâti. La reconversion des églises paroissiales fermées est abordée comme une pratique durable en lien avec les besoins locaux, face à la démolition qui apparaît comme une pratique controversée dans les logiques environnementales actuelles.

8 Les propos se fondent sur un travail de doctorat en architecture ${ }^{6}$ sur la région urbaine de Lyon Saint-Étienne. Suite à un inventaire des églises paroissiales ${ }^{7}$ et de leur usage, des premiers constats émergent, permettant de cerner les enjeux de démolition et de reconversion de ces édifices. 


\section{Développement durable et patrimoine : des concepts liés}

Alors que le développement durable fait référence à une idée aussi millénaire que «la lutte de l'homme pour sa survie dans la nature, d'un usage prudent et modéré des ressources » (Bartenstein, $2005: 292$ ), la définition la plus connue et utilisée aujourd'hui, date pourtant de 1987. Issue du rapport Brundtland, elle se formule ainsi : «Le développement durable, c'est s'efforcer de répondre aux besoins du présent sans compromettre la capacité de satisfaire ceux des générations futures ${ }^{8}$.

10 Cet énoncé constitue davantage un programme d'action qu'une définition. En ce sens, le développement durable se structure, depuis le Sommet de la Terre de Rio en 1992, par la conciliation de trois piliers indissociables: le développement économique, la qualité de l'environnement et la justice sociale. Dans cette logique, le principe d'une gestion économe, équitable et juste des ressources doit prendre en compte le territoire comme une véritable ressource (Stein, 2012). La mise en valeur de ses richesses, tant matérielles qu'humaines, passe par l'utilisation raisonnée du sol tout comme la récupération et le réemploi des constructions, en accord avec la communauté locale. Afin d'éviter le gaspillage et de réutiliser ce qui peut l'être, le patrimoine constitue une ressource territoriale non renouvelable qu'il s'agit de sauvegarder, d'économiser et de valoriser ${ }^{9}$. Grâce à ces actions, qui consistent à « faire durer dans le temps » des valeurs, « le patrimoine rejoint les intentions du développement durable » (Dris, 2012 : 14).

11 D'ailleurs, la référence au patrimoine, sa conservation et donc sa transmission aux générations futures est devenue le premier argument pour invoquer et légitimer la durabilité à l'échelle planétaire (Lazzarotti, 2003). En effet, l'évolution de la notion de patrimoine au-delà de la figure du monument historique et son élargissement au niveau européen et mondial (Gravari-Barbas, 2017) ont donné naissance à plusieurs textes de référence, et notamment à la convention de Budapest rédigée par l'Unesco en 2002. Elle s'engageait entre autres « à maintenir un juste équilibre entre la conservation, la durabilité et le développement, de façon à protéger les biens du patrimoine mondial grâce à des activités adaptées contribuant au développement social et économique et à la qualité de vie de nos communautés ${ }^{10}$ ". Ces notions permettent de raccrocher le patrimoine au développement durable et expriment une volonté « de mieux intégrer la dimension temporelle, de mieux articuler le passé, le présent et le futur de nos sociétés, dans une logique de transmission et de solidarité intergénérationnelle» (Garat et al., 2005: 2). Alors que certains évoquent une véritable tautologie (Garat et al., 2005) dans le fait de rapprocher développement durable et préservation du patrimoine bâti, et que les élus s'emparent de ce discours, la réalité n'est pas si évidente. En effet, «dans un contexte national de mesures de défiscalisation qui accentuent la spéculation immobilière " (Garat, et al., $2005: 14$ ), une logique de densification est mise en œuvre, pour contrer certes celle de l'étalement urbain, mais qui se fait au détriment de la conservation du bâti ancien. Cette dynamique tend à justifier la démolition d'héritages architecturaux jugés sans intérêt ou qualifiés de petits patrimoines, face à une sélection ponctuelle d'édifices dignes d'intérêt, bien souvent classés au titre des monuments historiques et mise en avant par les professionnels de la ville.

12 François Choay (1992) considère que la pratique du réemploi devrait faire l'objet d'une pédagogie particulière, car elle relève du bon sens, mais aussi d'une sensibilité inscrite 
dans la longue durée des traditions urbaines et des comportements patrimoniaux, propre à chaque territoire. Elle évoque le "rôle propédeutique » (Choay, 1992:198) du patrimoine bâti considéré comme un enseignement, un outil de transmission de savoir-faire via la restauration du bâti existant ou sa réutilisation qu'elle oppose à la muséification du bâti patrimonial, propre à l'héritage culturel français. Dans une logique de durabilité, de transmission aux générations futures, le patrimoine ainsi conçu pourrait conduire à la réactualisation d'activités artisanales multiples en lien avec les ressources, les connaissances et la main-d'œuvre présentes dans les territoires.

Alors que les églises, relevant du patrimoine religieux - et plus particulièrement du patrimoine cultuel - font consensus quant à leurs valeurs patrimoniale, historique et culturelle, leur pérennité est remise aujourd'hui en question par la baisse de la pratique et le manque de moyens humains et financiers de l'Église catholique comme des municipalités.

\section{Les églises paroissiales : quel patrimoine?}

Il est important de rappeler le contexte législatif en vigueur pour les églises paroissiales, car la particularité de leur devenir provient en partie de leur date de construction. En France, deux types de propriétaires existent, car il y a pour les églises un avant et un après 1905. De plus, un second régime juridique peut s'ajouter lorsqu'une église possède une protection au titre des monuments historiques.

\subsection{Les églises, des édifices cultuels}

\subsubsection{Avant 1905}

Depuis la loi de séparation des Églises et de l'État de 1905, et celle sur l'exercice public des cultes de 1907, toutes les églises construites avant 1905 appartiennent aux communes (Fornerod, 2013). Elles sont mises à la disposition des fidèles et des ministres du culte pour la pratique de leur religion. Ces édifices sont donc soumis à une affectation gratuite et exclusive.

En plus de cet usage unique, lors de la mise en vigueur de ces lois, les églises sont rentrées dans le domaine public en devenant propriétés communales ${ }^{11}$. Elles sont donc contraintes aux principes de la domanialité publique : l'inaliénabilité et l'imprescriptibilité.

Aujourd'hui, certaines municipalités, notamment rurales et peu peuplées, deviennent impuissantes face à ce patrimoine immobilier peu utilisé, voire pas entretenu. Après des années sans entretien, quand tombe le devis de la restauration, il apparaît parfois plus rationnel de démolir ces édifices même si, liés dès l'origine au développement de ces villages, ils en constituent la mémoire collective et individuelle (Maurey, 2015).

\subsubsection{Après 1905}

Quant aux églises construites après cette législation, elles sont propriété des diocèses. Depuis quelques décennies, par manque de prêtres et d'argent, des regroupements paroissiaux s'opèrent, condamnant à la fermeture des églises déjà en surnombre. Délaissées par l'usage religieux suite à ces réorganisations, ces constructions sont les édifices cultuels le plus en manque d'usage et menacés de démolition par suite d'une 
vente. Ces édifices sont d'autant plus en péril que leur forme et leur mise en œuvre modeste, bien qu'exemplaire, n'entrent pas dans les représentations patrimoniales reconnues. De plus, la valeur foncière des terrains est bien supérieure à la valeur immobilière du bâti. Stratégiquement pensée à l'époque de leur implantation, la situation de ces terrains demeure au cœur des dynamiques urbaines actuelles. Ces constructions subissent aujourd'hui, comme tout autre édifice non rentable, une pression immobilière accrue.

19 En d'autres termes, en France, la législation en vigueur sur les édifices cultuels, dont relèvent les églises communales, s'applique pour mener à bien une reconversion. Ces dispositions légales nécessitent dans un premier temps la désaffectation juridique du lieu et, si une commune veut vendre une église pour un projet privé, son déclassement du domaine public ${ }^{12}$. Gérées par le droit commun, les églises diocésaines, qui relèvent du domaine privé, ne sont pas soumises à une désaffectation juridique, mais le diocèse procède à une exécration ${ }^{13}$.

\subsection{Les églises, un patrimoine culturel}

En plus de leur usage cultuel, les églises sont largement reconnues comme un véritable patrimoine culturel. L'attribution de cette qualité concerne ici toutes les églises faisant l'objet d'une protection patrimoniale, qu'elles soient construites avant ou après 1905. Par protection patrimoniale, nous entendons tous les règlements qui protègent juridiquement ${ }^{14}$ les édifices d'une dénaturation ou d'une démolition. Aujourd'hui, il semble nécessaire de mettre en perspective cette législation historique des monuments historiques avec les nouvelles reconnaissances patrimoniales.

\subsubsection{Une reconnaissance étatique : les monuments historiques}

21 Comme le souligne Anne Fornerod, les « édifices cultuels représentent la composante la plus ancienne du patrimoine culturel et numériquement la plus importante des monuments historiques » $(2013: 27)$. En effet, d'après les derniers chiffres du ministère de la Culture et de la Communication (2017), sur les 44655 édifices classés et inscrits religieux, soit environ 13400 édifices. Parmi ces édifices religieux, seuls certains sont qualifiés d'églises. Ce corpus constitue historiquement un patrimoine reconnu par de véritables protections juridiques. Les édifices concernés par ces protections sont en grande majorité des propriétés communales ${ }^{15}$.

\subsubsection{Naissances d'autres reconnaissances patrimoniales}

Alors que les procédures de classement et d'inscription sont en baisse depuis les années $1990^{16}$, d'autres moyens de valorisation et de préservation se déploient comme le label «Architecture contemporaine remarquable ${ }^{17}$ » avec la loi relative à la liberté de la création, à l'architecture et au patrimoine. Cette reconnaissance ou valorisation patrimoniale n'est pas une protection, au sens de l'imprescriptibilité que procure le classement ou l'inscription au titre des monuments historiques. Cependant, les nouvelles prescriptions d'application du label apportent quelques contrôles et restrictions ${ }^{18}$.

23 Ce label peut concerner les églises de moins de cent ans d'âge, soit les églises propriétés des diocèses. Comme mentionné ci-dessus, elles relèvent d'un régime juridique de droit commun, donc moins protecteur vis-à-vis de ce patrimoine qui est aujourd'hui le plus 
sujet à une aliénation et dont, symboliquement, l'architecture incarne moins la figure patrimoniale aimée et attendue d'une église ${ }^{19}$ (Perrin, 2005).

Les éléments bâtis à préserver (EBP) constituent un autre outil de conservation en lien avec le plan local d'urbanisme (PLU). Le PLU se fonde sur un Projet d'Aménagement et de Développement Durable (PADD) et détermine les conditions qui permettent d'assurer un équilibre entre trois volets : la qualité urbaine, architecturale et paysagère, la diversité des fonctions urbaines et rurales comme la mixité sociale dans l'habitat, et la réduction de la pollution de notre environnement en maîtrisant et favorisant des sources énergétiques renouvelables. Le premier volet comprend un aspect patrimonial en mentionnant « la sauvegarde des ensembles urbains et du patrimoine bâti remarquables »

Á travers les spécificités de son territoire répercutées dans son PLU, «n'importe quelle commune ou intercommunalité peut décider d'injecter une réelle ambition patrimoniale dans sa planification urbaine ". Ce règlement peut ainsi jouer le rôle de conservation des éléments identifiés comme patrimoniaux en localisant les « immeubles, espaces publics, monuments, sites et secteurs à protéger, à mettre en valeur ou à requalifier pour des motifs d'ordre culturel, historique, architectural ou écologique ". Au même titre que certaines zones reçoivent des orientations urbaines, le PLU établit pour les EBP - que ce soit un bâtiment, une façade ou un élément architectural - « les prescriptions de nature à assurer leur préservation ». Cette disposition d'urbanisme peut s'appliquer à toutes les églises, qu'elles soient communales ou diocésaines, qui ne font pas déjà l'objet d'une protection au titre des monuments historiques. En revanche, une église peut cumuler en étant à la fois un EBP et avoir le label « Architecture contemporaine remarquable ».

Même si la lourdeur du contexte juridique qui encadre le corpus des églises - constitué parfois d'une superposition de régimes différents - est souvent avancée comme un frein à leur reconversion, les situations de transformation sont entièrement prévues par les lois.

\section{Premiers constats d'une recherche}

27 L'inventaire mené sur les églises paroissiales de la région urbaine Lyon Saint-Étienne permet rapidement de cerner les enjeux de reconversion et de démolition. Sur l'ensemble des 427 édifices inventoriés (Figure 1), on dénombre 11 églises démolies depuis les années $1950^{20}$, et 3 vont l'être d'ici fin 2018. Soit un total de 14 édifices démolis (3,3\%), contre 26 reconvertis $(6,0 \%)$ depuis 1905 , suivant un large panel de nouveaux usages: logements, maison de l'enfance, cinéma, bibliothèque, mairie, salles d'escrime, école, centre artistique et culturel. Parmi ces reconversions, 4 sont dites mixtes (partage du culte avec un autre usage permanent, autre qu'évènementiel) et 22 totales. 
Figure 1. Inventaire par usages des églises et chapelles paroissiales de la région urbaine Lyon Saint-Étienne

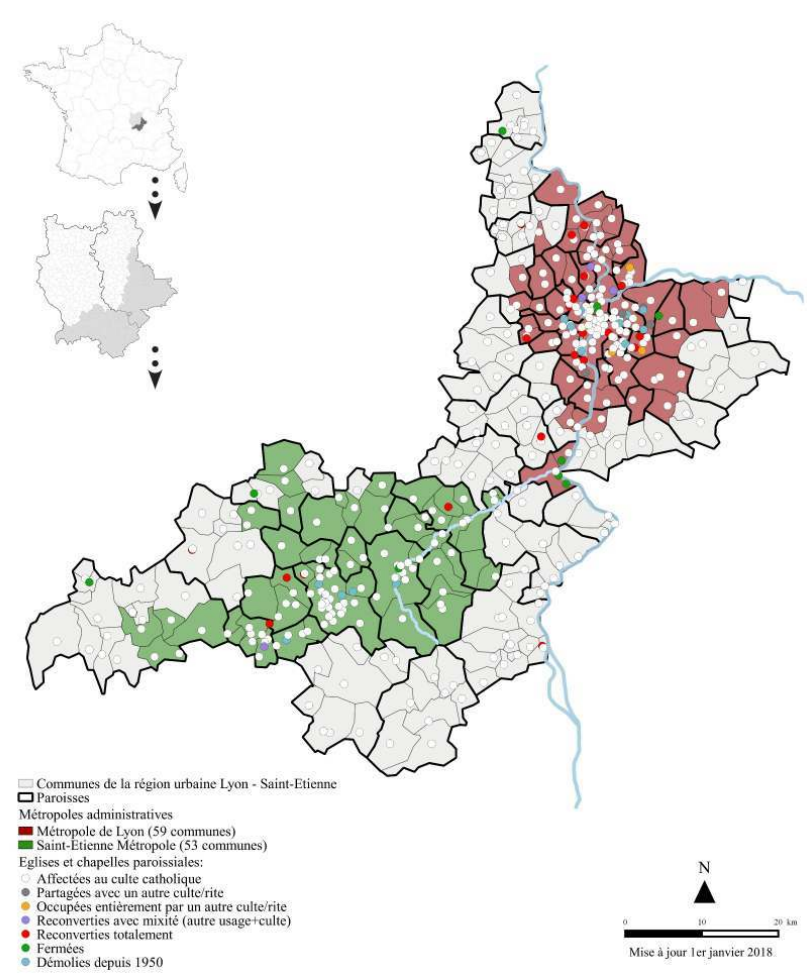

Cartographie : Mélanie Meynier-Philip, 1er janvier 2018

De plus, l'étude des diverses situations de mutation du bâti (fermeture, vente, reconversion, démolition) a fait émerger trois catégories d'églises en fonction de leur date de construction, de leur propriétaire, de leur situation géographique et de leur reconnaissance patrimoniale. La première catégorie regroupe les " églises historiques ", généralement à l'origine d'un îlot urbanisé en milieu urbain ou rural. Propriétés des communes, elles représentent la figure type d'une église avec une nef et un clocher, bien souvent protégées, classées ou inscrites, au titre des monuments historiques. La deuxième catégorie est celle des " églises XIX ${ }^{\mathrm{e}}$ » bâties en grande quantité à cette époque, et parfois en remplacement d'églises primitives devenues trop petites. Situées en milieu urbain comme rural, propriétés des communes pour la plupart, elles ne font pas, ou très rarement, l'objet de reconnaissance patrimoniale. Enfin, les «églises $\mathrm{xx}^{\mathrm{e}}$ » ou «églises modernes » constituent la troisième catégorie. Propriétés privées détenues par les diocèses, elles se localisent en périphérie des grandes villes dans les zones urbaines développées pour répondre à l'expansion démographique qui suivit la Seconde Guerre mondiale. Sauf exception, ces édifices manquent de reconnaissance patrimoniale tant auprès des experts du patrimoine ${ }^{21}$ que de l'opinion publique.

\section{1. État des lieux des églises démolies}

Les églises construites au cours du $\mathrm{XIX}^{\mathrm{e}}$ siècle et durant la seconde moitié $d u \mathrm{xx}^{\mathrm{e}}$ siècle comptent parmi les types d'édifices les plus menacés, compte tenu de leur faible reconnaissance architecturale et donc de leur moindre protection patrimoniale ${ }^{22}$. Parmi les édifices démolis sur le territoire d'investigation: un édifice historique, l'ancienne 
chapelle Saint-Alban (Lyon $8^{\mathrm{e}}$ ) pourtant inscrit au titre des monuments historiques en $1936^{23}$, cinq avaient été construits au XIX ${ }^{\mathrm{e}}$ siècle, comme l'église Sainte-Marie de l'Assomption à La Mulatière ou l'ancienne église du Point du Jour à Lyon, contre cinq après la Seconde Guerre mondiale, comme l'église Sainte-Bernadette à Saint-Étienne ou l'église Saint-Pierre-Chanel à Villeurbanne. L'inventaire révèle trois autres églises modernes, construites après 1950, aujourd'hui fermées, sans usage et en attente d'une démolition proche: l'église Notre-Dame de l'Espérance (1956-1966, Pierre Genton) à Villeurbanne, et dans la Loire l'église du Bon Pasteur (1957-1959, Jean Farat) au ChambonFeugerolles (Figure 2) ainsi que l'église Notre-Dame de l'Annonciation, dite église de la Baraillière (1963-1966, Raymond Aillaud et Louis Vendange) à Saint-Jean-Bonnefonds. Si les médias s'emparent volontiers des controverses ${ }^{24}$ qui émergent en cas de menace de démolition ou de démolition effective, on observe une forte mobilisation des citoyens, qu'ils soient croyants ou non. En effet, les paroissiens, autrement appelés la communauté d'usage $^{25}$, sont rapidement rejoints par les habitants du quartier et de la ville pour organiser leurs revendications en s'impliquant dans la sphère publique. Via la parole d'associations lançant des pétitions contre les démolitions, menant des actions dans les conseils de quartier, cette communauté patrimoniale forme une véritable ressource territoriale pour envisager et entreprendre, en accord avec les besoins et la population, un nouvel usage.

Figure 2. Église du Bon Pasteur au Chambon-Feugerolles (42), en attente de démolition

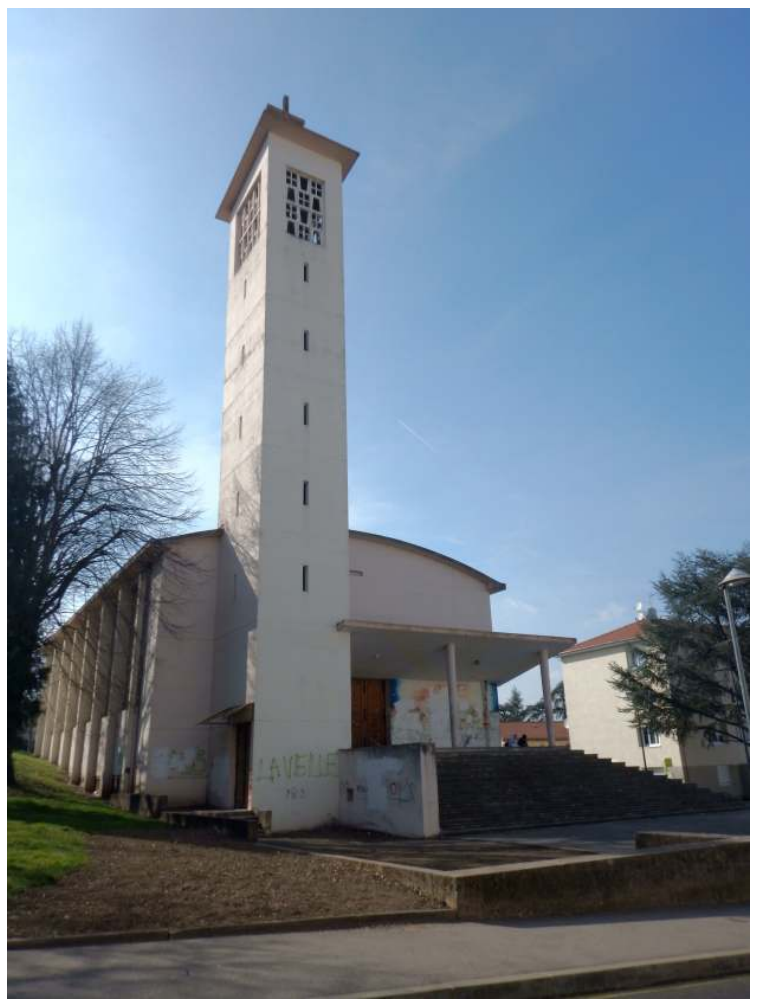

Photo : Mélanie Meynier-Philip, 15 mars 2016 


\section{2. État des lieux des églises reconverties}

Les églises reconverties sont principalement des « églises historiques », au nombre de 12, et des " églises modernes ", au nombre de 11, dont 7 construites après 1950. Seulement 3 églises construites au XIX siècle sont à ce jour transformées, l'ancienne église de la Saulaie à Oullins, l'ancienne chapelle de la Buissière à Rillieux-la-Pape et l'église SaintCamille à Vaise dans le $9^{e}$ arrondissement de Lyon, qui partage sa nef entre le culte catholique orthodoxe et une épicerie solidaire (Figure 3).

Figure 3. Église Saint-Camille à Lyon (69), la moitié de la nef accueille une épicerie solidaire

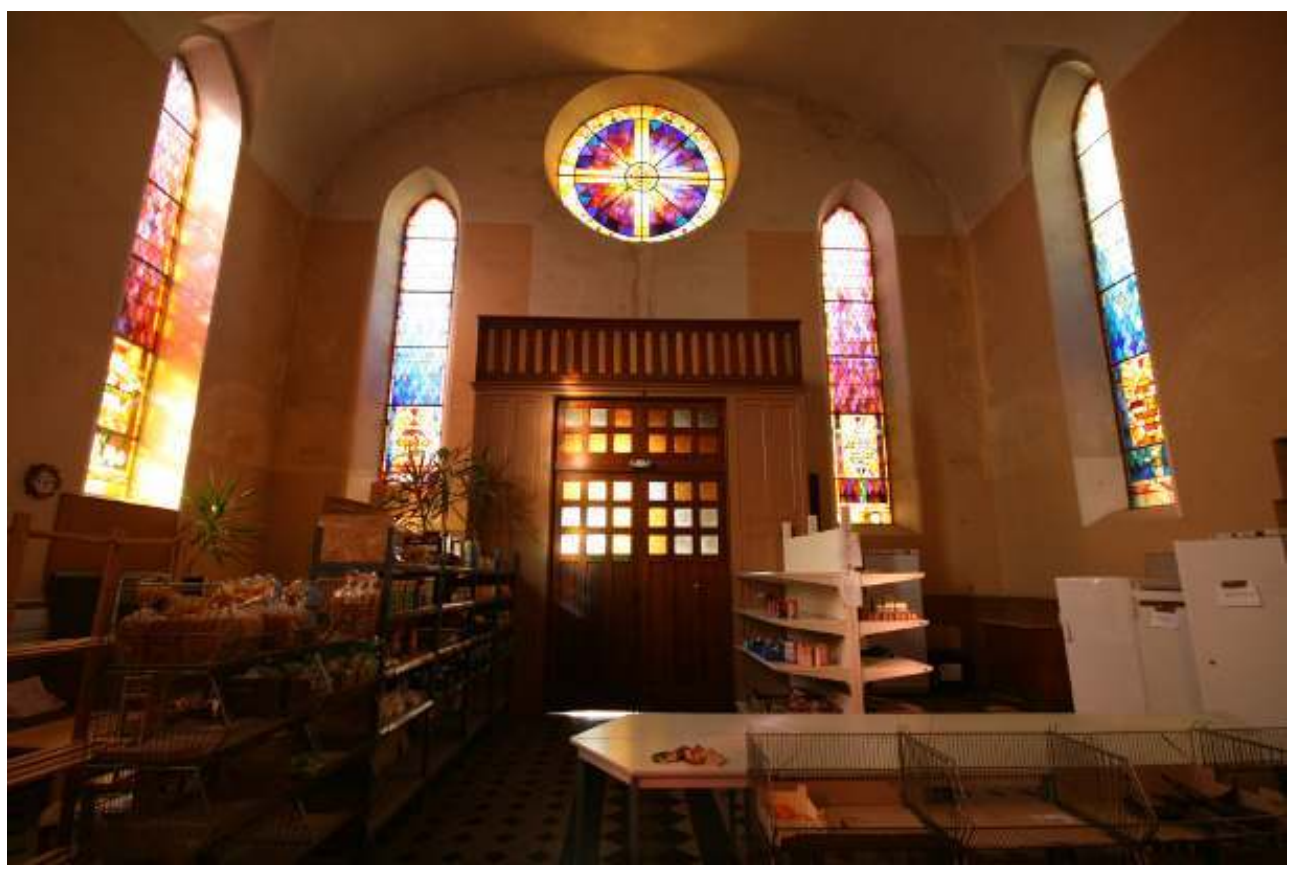

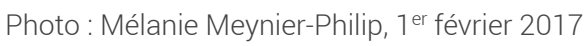

31 Dans le premier cas de figure, les bâtiments, souvent protégés patrimonialement, sont insérés dans le tissu urbain d'origine. C'est le cas de l'ancienne chapelle Sainte-Catherine à Saint-Marcellin-en-Forez, dont le clocher a été inscrit au titre des monuments historiques en 1978 et qui est reconvertie en médiathèque depuis 1998 (Figure 4). L'ancienne église Saint-André à Villars a également été transformée en salle des mariages et des conseils de la mairie en 2000. Une fois le culte retiré, ces édifices se sont naturellement prêtés à un autre usage au cœur du village. Leur réemploi développe et transmet le savoir-faire local par la restauration d'une charpente, d'une toiture ou d'un mur en pisé comme à Saint-Marcellin-en-Forez.

Dans le second cas de figure, les églises modernes affichent un fort taux de reconversion. D'une part leur implantation a été stratégiquement pensée ${ }^{26}$ dans une volonté de maillage territorial (Chalabi, 2009) et elles demeurent encore aujourd'hui au centre des quartiers. D'autre part, leurs caractéristiques constructives, de type plan libre, font d'elles des manifestes de flexibilité architecturale ${ }^{27}$, comme le montre la transformation en cinéma de l'ancienne église de Balmont à la Duchère ou encore l'ancienne église SainteGeneviève, transformée en salle d'escrime à Bron (Figure 5). 
Figure 4. Médiathèque de Saint-Marcellin-en-Forez (42) ancienne chapelle Sainte-Catherine

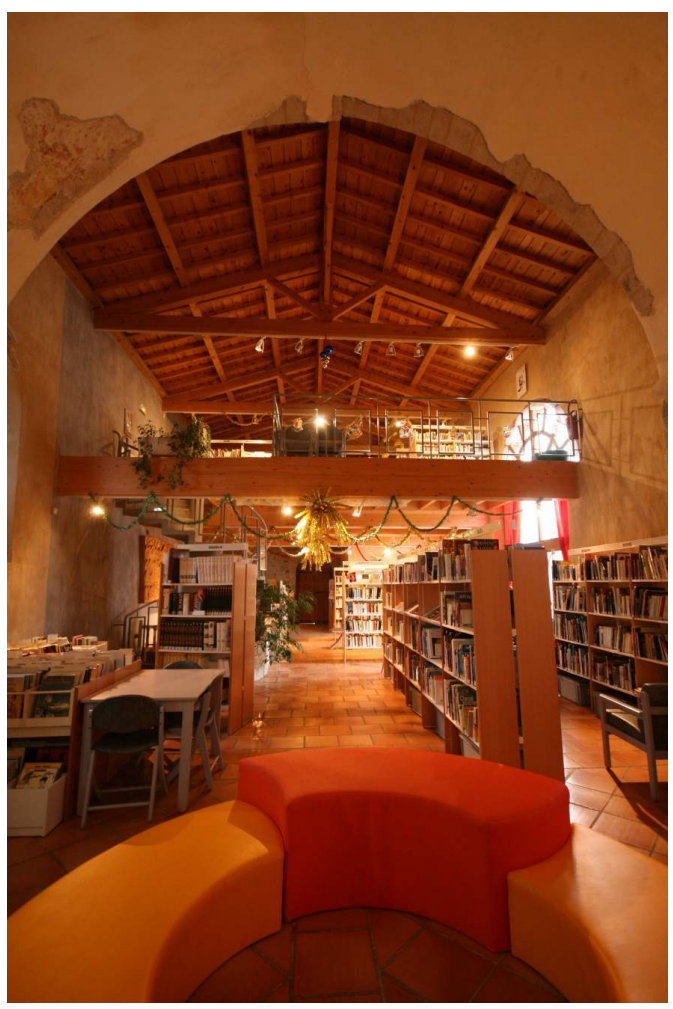

Photo : Mélanie Meynier-Philip, 13 janvier 2017

Figure 5. Salle d'escrime de Bron (69) ancienne église Sainte-Geneviève

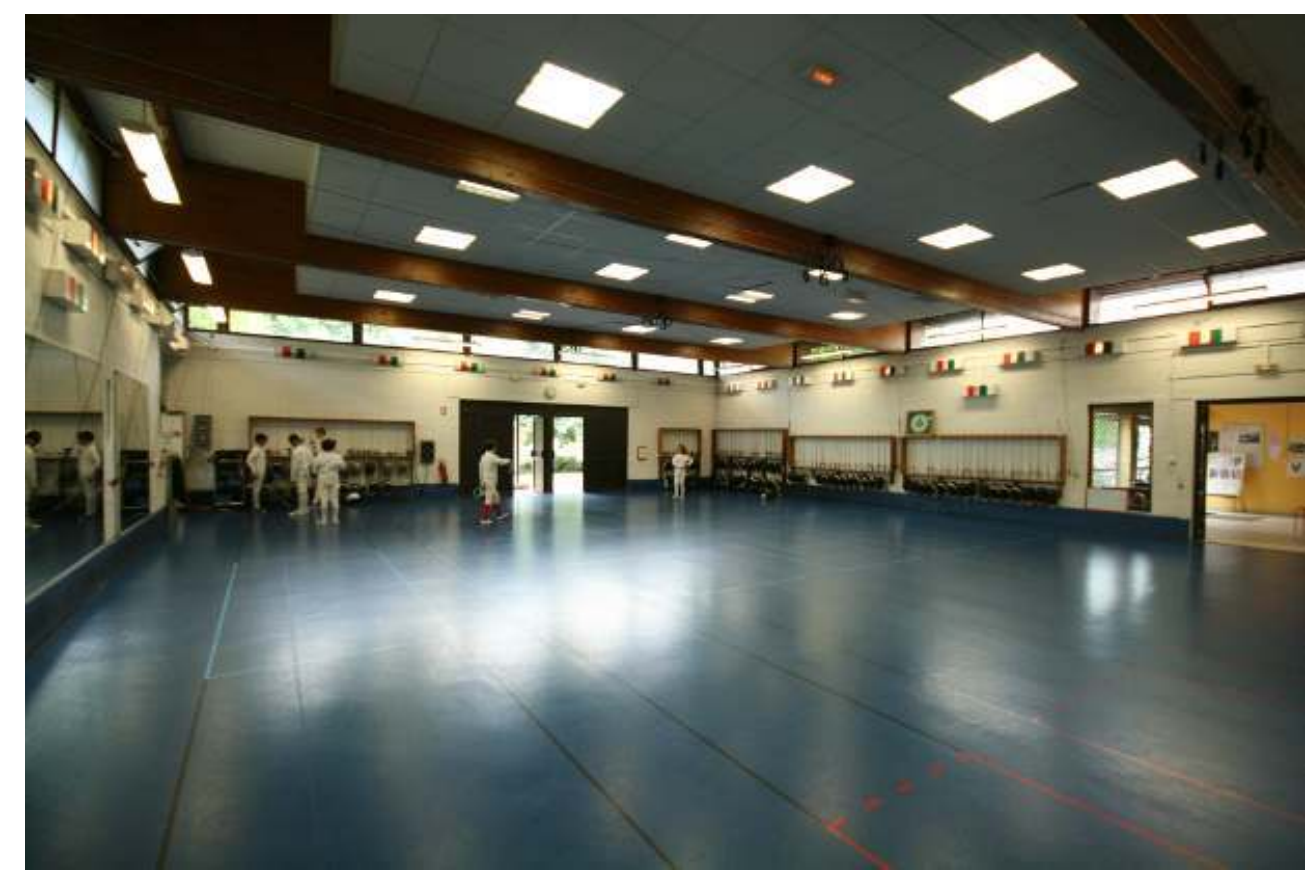

Photo : Mélanie Meynier-Philip, 28 juin 2017 


\section{La démolition et ses enjeux}

Dès 2012, l'annonce de la vente pour destruction de l'église du Cœur Immaculé de Marie à Villeurbanne (Figure 6) a révélé, via l'association Cadre de vie et patrimoine, des habitants qui étaient «contre " la démolition de l'édifice ${ }^{28}$, mais "pour » le projet qui envisageait un immeuble de logements sociaux et un centre d'hébergement et de réinsertion sociale ${ }^{29}$. Les paroissiens, premiers informés de cette mutation, se sont rapprochés de l'association, elle-même impliquée dans le conseil de quartier, afin de prendre part au débat et de faire entendre leurs revendications. Malgré cette mobilisation, qui a également pris la forme d'une pétition ${ }^{30}$ (553 signatures) et d'articles sur le site internet de l'association ${ }^{31}$, la mairie, en accord avec le diocèse propriétaire, n'a pas entrepris des démarches de consultation. Pourtant, les ressources humaines présentes à travers la mobilisation des habitants autour de ce projet offraient une véritable opportunité démocratique. Ce groupe de citoyens, nommé aussi la " communauté patrimoniale », est défini et légitimé par la convention de Faro comme des "personnes qui attachent de la valeur à des aspects spécifiques du patrimoine culturel qu'elles souhaitent, dans le cadre de l'action publique, maintenir et transmettre aux générations futures ${ }^{32}$ ». Elle s'est ici manifestée pour conserver d'une part, l'ancienne chapelle construite en 1838 comme dernière trace du château de la Ferrandière ${ }^{33}$ et d'autre part, le bâtiment réceptacle de la mémoire individuelle et collective des habitants du quartier. Les actions menées par la communauté ont tout de même porté leurs fruits. En effet, même si la ville n'était pas l'initiatrice du projet, face au mouvement et à la demande citoyenne, elle a finalement arbitré cette opération à travers la mise en place d'un programme de logements sociaux par un Office public d'Habitations à loyer modéré (HLM), Est Métropole Habitat dont le président est aussi le Maire de Villeurbanne. Cette mobilisation a permis d'éviter la vente de l'ancienne église à un promoteur privé et une démolition totale. 
Figure 6. Démolition de l'église du Cœur Immaculé de Marie à Villeurbanne (69)

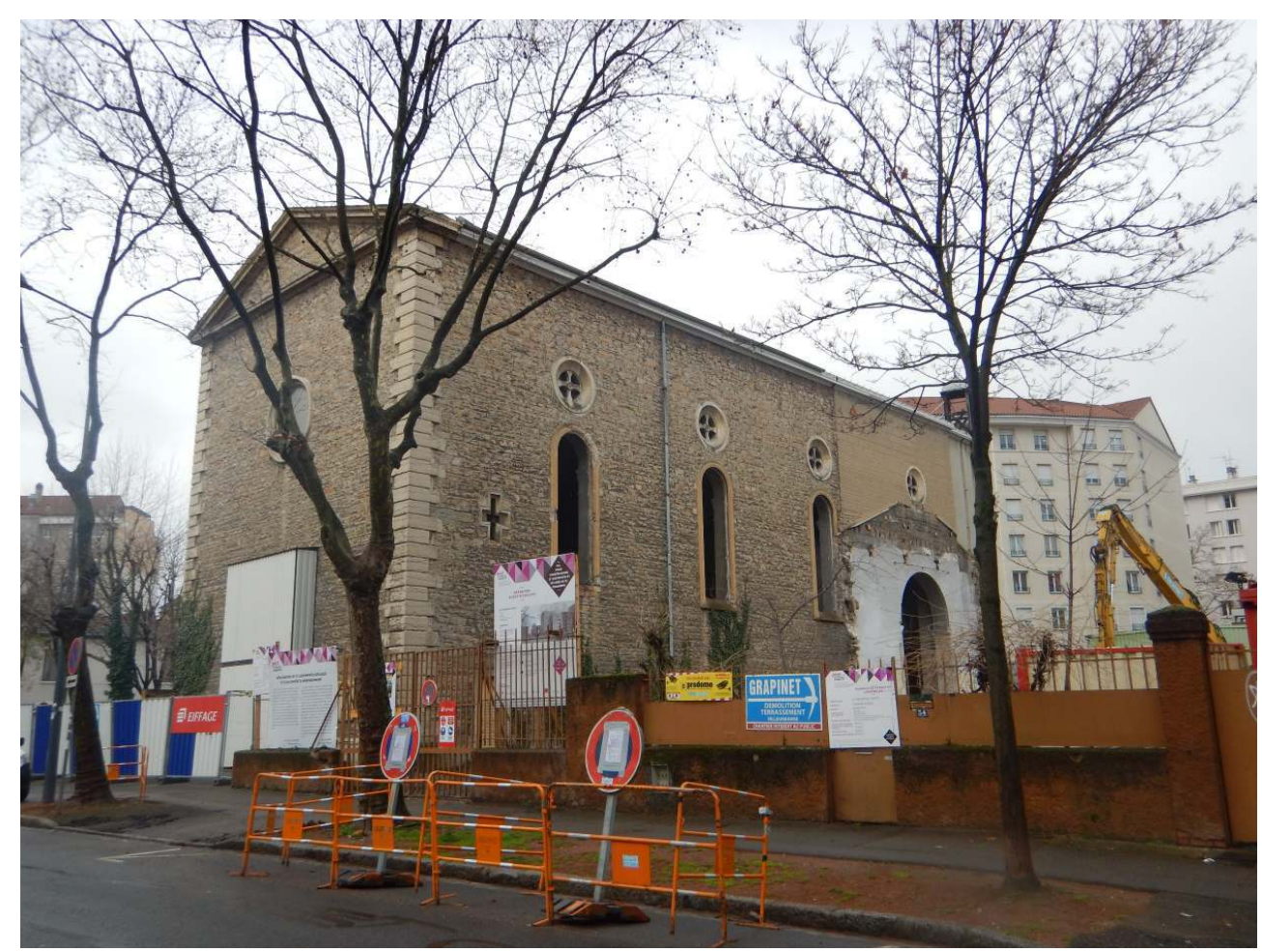

Photo : Mélanie Meynier-Philip le 03 mars 2016

34 La disparition d'un patrimoine, entraînée par l'acte irréversible de la démolition, ne semble pas en phase avec la volonté affichée des villes actuelles qui se revendiquent durables, alliant équilibre écologique, en répondant à la question de la gestion des déchets, et revendication sociale, en prenant en considération la mobilisation patrimoniale, véritable «mobilisation politique» aux enjeux démocratiques (Veschambre, 2008 : 74). En ce sens, dans le contexte villeurbannais qui n'échappe pas au discours de ville durable, l'agence d'architecture A-MAS en charge du projet du centre d'hébergement et de logements sociaux, a tenté de concilier mémoire, par une démolition partielle et développement durable, par un réemploi volontaire de certains éléments.

\subsection{Démolition partielle}

Suite aux revendications de la communauté locale contre la disparition de l'ancienne église, les pré-études menées par les maîtres d'ouvrage formulaient la conservation « des façades visibles depuis la rue ${ }^{34} »$ comme un des enjeux du projet. Cependant, dès le début des esquisses, l'architecte sélectionné avait alerté sur le fait que les objectifs environnementaux attendus dans l'opération ne permettaient pas d'installer des logements dans le volume existant ${ }^{35}$. Après deux propositions, le choix s'est orienté vers un arasement de l'église à 4,80 mètres de hauteur, pour maintenir l'emprise au sol du lieu. La formule "le plein sera désormais le vide ${ }^{36}$ » exprime la volonté de conserver une trace en négatif d'un édifice qui, malgré les profondes transformations du quartier au cours du $\mathrm{xx}^{\mathrm{e}}$ siècle, avait perduré comme un élément fixe du paysage urbain. Aujourd'hui ce vide, délimité par des murs tronqués, est la base autour de laquelle le nouveau projet s'organise. Si la forme en plan est maintenue par ces restes de murs, la figure en hauteur, 
autrement dit le volume et la façade urbaine de l'église qui sont des «éléments constitutifs de la figure ecclésiale » (Noppen, 2006 : 285) ont disparu pour laisser place à ce vide invoqué par le concepteur. Dans ce cas, même si la démolition n'a pas été totale, elle a néanmoins, dans un premier temps, été vécue comme telle par les habitants. L'attitude architecturale radicale, une quasi « décapitation ", vis-à-vis de ce patrimoine villeurbannais a d'abord choqué les riverains. Selon le maître d'œuvre, cette solution a été entraînée par les normes environnementales exigées par la nouvelle opération ${ }^{37}$. L'installation de logements dans le volume de la nef sans modification des façades ne permettait pas de proposer des espaces habitables avec un taux d'ensoleillement digne et réglementaire.

\subsection{Recyclage volontaire : « La mémoire renouvelée »}

Dans cet exemple, au-delà des réglementations écologiques attendues dans les nouveaux logements, le choix a été orienté vers le réemploi de certains éléments, permettant également une continuité mémorielle du site. Ainsi, les matériaux issus de la déconstruction ont été en partie réutilisés dans la construction actuelle, comme "une manière d'inscrire le nouveau projet dans une continuité historique, tout en apportant une qualité et pérennité par les matériaux ${ }^{38}$ ». Le regard des habitants s'est finalement transformé, en voyant dans cette démarche un certain respect et une valorisation de ce bâti ancien, face à une démolition qui aurait pu être totale. Ainsi, les pierres ont été utilisées comme pierres banchées en rez-de-chaussée pour reformer les murs arasés de l'église formant une cour et un élément sculpté a été apposé sur la nouvelle façade de l'immeuble de logements. Le sol intérieur de la nouvelle cour reprend le tracé du dallage de l'ancien lieu de culte. Dans une logique de recyclage, les tuiles de la couverture devaient ${ }^{39}$ être récupérées et concassées sur place pour protéger l'étanchéité des toitures-terrasses. Pour des raisons financières, cela n'a pas pu être fait.

Les déchets restants générés par la démolition forment cependant des reliquats et constituent un surplus de matière que notre milieu de vie, la planète, ne peut gérer naturellement ${ }^{40}$.

Figure 7. Vues extérieure et intérieure des traces de l'ancienne église du Cœur Immaculé de Marie à Villeurbanne (69) après construction du centre d'hébergement et de logements sociaux
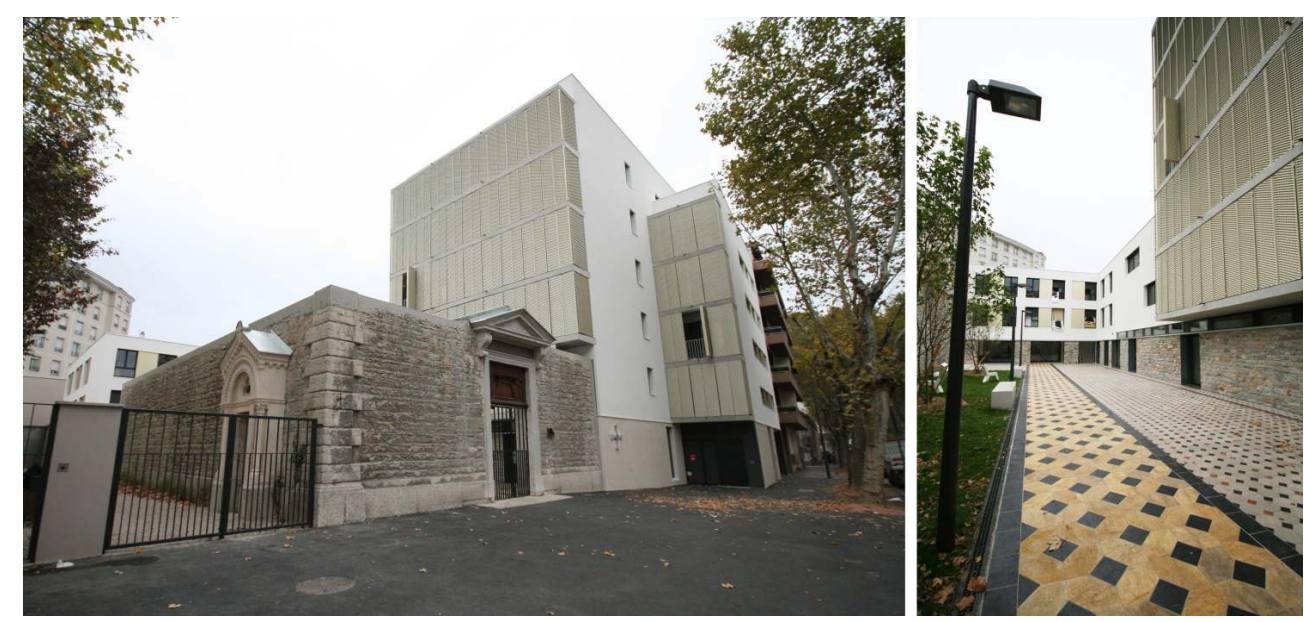

Photos : Mélanie Meynier-Philip, 29 octobre 2017 
Dans cette optique de durabilité, même si on tente de traiter au maximum ces restes par un nouveau cycle de vie de la matière par le recyclage, Jean-Marc Huygen distingue cette pratique du réemploi. Il pense qu'il faut privilégier d'abord la réutilisation par du réemploi ${ }^{41}$ de matériaux et d'éléments de bâtis avant d'envisager le recyclage. Il démontre que cette attitude responsable, qui permet "une économie de la matière déjà transformée (exploitée, ayant utilisé de l'énergie et devenue trace de culture)» (Huygen, 2008 : 13), enrichit également le nouvel objet ou bâti d'une « composante patrimoniale » car l'objet réemployé " garde sa mémoire, l'histoire de ce qu'il a vécu, [...], c'est un héritage » (Huygen, 2008:12). Ces pratiques doivent se généraliser et permettre de reconstruire des édifices à partir d'autres déconstruits (Namias, 2016). Dans cette lignée de pensée, au niveau national ${ }^{42}$, la démarche de "construire autrement" menée par l'architecte Patrick Bouchain est éclairante (Bouchain, 2006). En s'appuyant sur la spécificité du contexte, il tente d'allier demande sociale, forces humaines et matières en place pour réaliser un projet qui, au regard de l'histoire du site, a du sens aujourd'hui et laisse place aux mutations de demain. Afin de minimiser leur impact écologique et social, il utilise dans ses projets à la fois les matériaux et les humains présents sur le site.

Par ailleurs, l'observation des matériaux issus de la démolition d'églises démontre qu'il s'agit de matières aisément réemployables. Le gros œuvre est majoritairement récolté comme des pierres de taille (matière noble) parfois des briques ou du béton pour les églises modernes, des poutres en bois ou en métal issues de la charpente et des tuiles. Les matières dites de second œuvre sont du plâtre formant la voûte et surtout du mobilier, tel que les bancs présents en grand nombre, mais facilement transposables dans un autre lieu, comme un intérieur pour les assises en bois ou un espace public pour les bancs en béton des églises modernes ${ }^{43}$. La particularité des ressources issues de la démolition des églises est qu'elles offrent de grandes possibilités de réemploi permettant une continuité mémorielle, paramètre important dans le contexte de ce corpus et de sa patrimonialisation, alliée aux préoccupations environnementales. En dehors de l'exemple villeurbannais, l'étude menée sur la région urbaine Lyon Saint-Étienne démontre que les dernières démolitions d'églises menées depuis 1950 n'ont laissé aucune trace sur l'espace public. La tabula rasa est souvent privilégiée par les diocèses ${ }^{44}$ qui, contraints par des raisons pratiques et économiques, ferment et vendent les églises qui leur appartiennent à des promoteurs privés. Entre malaise face aux mobilisations patrimoniales que suscitent de tels édifices et parfois manque de connaissance de ce corpus, une nouvelle construction prend la place de l'ancien lieu de culte, sans marques ou artefacts (Veschambre, 2008), sans même de panneau ou plaque mémorielle explicative.

40 En comparaison des démolitions communément pratiquées, autrement dit sans réemploi systématique, regardons maintenant les enjeux de la reconversion qui rejoignent finalement le travail de Bouchain dans sa volonté de faire avec les paramètres locaux.

\section{La reconversion et l'échelle locale}

41 Les exemples de reconversion d'églises sur la région urbaine de Lyon Saint-Étienne montrent à quel point les bâtiments églises ont tout pour convenir à un projet de transformation en lien avec les pratiques, les besoins et les usages locaux.

42 L'église rurale se situe généralement sur la place principale du village, quant aux églises urbaines, elles sont idéalement placées à proximité d'un espace public et possèdent 
souvent en zone périurbaine un espace extérieur permettant l'accueil d'évènements paroissiaux. Ces terrains offrent en général de grands parkings facilement transformables dans le cadre d'un nouvel usage. En ce sens, les parcelles des églises sont des ressources, des réserves foncières pour développer des espaces publics et/ou verts comme des places, des parcs ou des jardins, aménagés en lien avec les besoins du quartier et de la nouvelle fonction. Dans un contexte urbain, alors qu'une réserve foncière est envisagée comme un levier pour densifier davantage, ces parcelles apparaissent comme de véritables réserves d'espaces verts et/ou d'espaces publics, à aménager pour la population et l'agrément du cadre de vie locale.

Figure 8. Maison de l'enfance de la Duchère à Lyon (69) ancienne église du Château

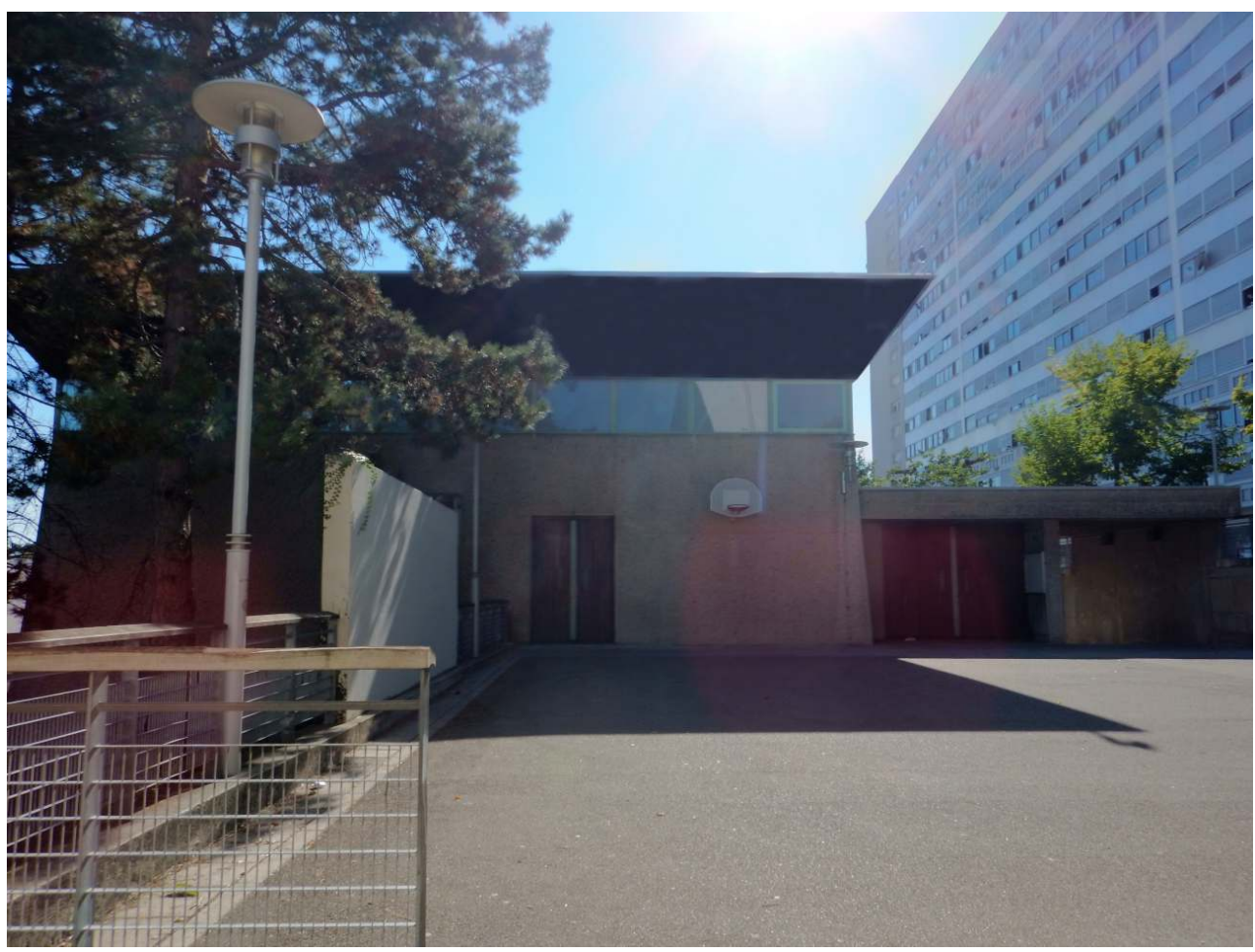

Photo : Mélanie Meynier-Philip, le 29 septembre 2016

La transformation à Lyon de l'église du Château (1960-1964, Maurice Novarina) en Maison de l'enfance de la Duchère (Figure 8), en 1981, en est un bel exemple. Insérée dans un quartier de barres de logements sociaux, à proximité des écoles, l'église construite dans les années 1960 s'est, en fonction des besoins de son environnement, transformée en centre d'accueil pour les enfants du quartier. Dans le cadre d'opérations de rénovations urbaines, vers les années 2000, un réaménagement a été entrepris sur le secteur. Telle une place, le parvis de l'église s'est ouvert aux autres espaces publics. Dédié aux activités du centre, cet espace reconfiguré en un terrain de sport ouvert augmente la continuité piétonne. Le foncier et le nouvel usage ont simplement renforcé la place de cet édifice dans son environnement, que ce soit par la pratique du lieu, l'accueil des jeunes, ou les espaces publics qu'il offre. Dans une zone en mutation, plutôt que de subir la démolition du bâtiment pour densifier la parcelle, la réserve foncière a été mise à profit pour l'intérêt collectif. 
caractéristiques des églises - mémoire individuelle et collective, centralité, réserve foncière ou réserve d'espaces publics/verts, ressource humaine à travers les mobilisations, transmissions de savoir-faire par le maintien du bâti -, les municipalités comme les diocèses doivent œuvrer de concert pour mener le processus de patrimonialisation attendue par les communautés locales et patrimoniales vis-à-vis d'un tel bâti. Que ce soient les associations ou les services municipaux qui œuvrent, le potentiel et la grande variété des projets de reconversion de ces édifices ont tout pour répondre aux enjeux de développement durable auxquels nous sommes confrontés. Face à des menaces de démolition, les églises délaissées par le culte, en quête d'un nouvel usage, pourraient et devraient être davantage envisagées comme un outil de développement local, tel que pratiqué au Québec ${ }^{46}$. En effet, dans cette province un projet novateur, nommé le «Plan églises », est mené grâce aux travaux de l'équipe de la chaire de recherche du Canada en patrimoine urbain, et dirigés par les spécialistes du patrimoine religieux Lucie K. Morisset et Luc Noppen. Cette action collective, semble être une réponse au problème de la fermeture des églises en exprimant à la fois de l'« empathie » (Letourneau, 2005 : XI) et une certaine "préoccupation » pour la cause du passé et celle du présent, pour celle des ancêtres et celle des contemporains, celle d'un projet qui actualise le patrimoine en liant passé et présent dans une réflexion tant théorique que pratique rejoignant les enjeux et le programme d'action du développement durable. Ce programme de recherche se veut comme une stratégie de reprise mise en œuvre à l'échelle d'un territoire (une ville, une région, un diocèse) par les acteurs du milieu universitaire, culturel, politique et religieux ${ }^{47}$.

L'idée d'un «Plan églises $»^{48}$ est née de l'observation et de la connaissance de tout ce qui a pu se faire jusqu'à maintenant au Québec, en matière de mutation d'églises, les réussites comme les échecs. L'expérience, et notamment le projet pilote de requalification de l'ancienne église Sainte-Brigide de Kildare (Noppen, 2008, 2013) à Montréal ${ }^{49}$, ont permis à la chaire de penser un projet de reprise à une échelle plus grande. Souvent, par manque 
de planification, d'encadrement, de concertation et de moyens techniques et financiers, des projets mettent en péril le patrimoine architectural et artistique religieux. Le processus de reconversion d'une église est complexe, long, et requiert diverses expertises qui impliquent un investissement de nombreux acteurs de différents secteurs. Alors que la reconversion d'une église peut s'avérer une belle opportunité de développement local et de mise en valeur pour des municipalités, celles-ci sont souvent mal outillées lorsque se présente un tel projet.

Afin d'éclairer et de faire avancer cette problématique d'églises en manque d'usage qui n'échappe pas au contexte français, le territoire de la région urbaine Lyon Saint-Étienne est questionné dans notre étude par ce référentiel et cette méthode québécoise à travers une recherche-action. Ce programme de recherche par le projet d'architecture donnera, on l'espère, des résultats dans les années à venir, car il y a là un réel défi pour les prochaines décennies.

\section{BIBLIOGRAPHIE}

Bartenstein K., 2005, « Les origines du concept de développement durable », Revue juridique de l'environnement, vol. 30, $\mathrm{n}^{\circ}$ 3, p. 289-297.

Bouchain P., 2006, Construire autrement : Comment faire?, Arles, Actes Sud, coll. «L'impensé ».

Chalabi M., 2009, «Les églises paroissiales construites dans la seconde moitié du xxe siècle et leur devenir : l'exemple de Lyon (Rhône) ", In Situ Revue des patrimoines, n 11, http:// insitu.revues.org/5887, (consulté le 17 février 2018).

Choay F., 1992, L'allégorie du patrimoine, Paris, Seuil, coll. « La couleur des idées ».

Cieren P., 2014, « Réflexion sur la reconversion des lieux de culte au cours de l'histoire », La Pierre d'Angle architecture, urbanisme, environnement, Recyclage des lieux de culte, n 65, p. 28-33.

Coomans T., Morisset L.K., Noppen L., 2006, Quel avenir pour quelles églises ? What future for which churches ?, Québec, Presses de l'université du Québec, coll. «Patrimoine urbain ».

Coomans T., Sauvé J.S., 2014, Le devenir des églises, Patrimonialisation ou disparition, Québec, Presses de l'université du Québec, coll. « Nouveaux patrimoines ».

Desmoulins-Hémery S., Palouzié H., 2006, « Regards sur les églises de France : lieux de culte, lieux de culture ", Actes du colloque de l'Association des conservateurs des antiquités et objets d'art de France, Alençon, du 13 au 15 octobre 2005, Arles, Actes Sud, 205 p.

Dris N. (dir.), 2012, Patrimoines et développement durable. Ressources - Enjeux - Lien social, Rennes, Presses universitaires de Rennes, coll. « Espaces et territoires».

Duboscq B., Moulinier P., 1987, Églises, chapelles et temples de France : un bien commun familier et menacé, ministère de la Culture et de la Communication, direction de l'administration générale et de l'environnement culturel, département des études et de la prospective, Paris, La Documentation française. 
Faltrauer C., Martin P., Obadia L., 2013, Patrimoine religieux : désacralisation, requalification, réappropriation : le patrimoine chrétien, Paris, Riveneuve, coll. « Actes académiques.

Fornerod A., 2013, Le régime juridique du patrimoine religieux, Paris, L'Harmattan, coll. « Droit du patrimoine culturel et naturel ».

Garat I., Gravari-Barbas M., Veschambre V., 2005, « Préservation du patrimoine bâti et développement durable : une tautologie ? Les cas de Nantes et Angers », Développement durable et territoires, Dossier 4, http://developpementdurable.revues.org/4913 (consulté le 27/02/2018).

Gravari-Barbas M., 2017, « Le patrimoine, de l'objet à la relation », in Barrère C. et al. (dir.), Mémoires et patrimoines, Des revendications aux conflits, Paris, L'Harmattan, coll. « Habitat et sociétés ", p. 41-49.

Hervieu-Léger D., 1999, Le pèlerin et le converti, La religion en mouvement, Paris, Flammarion, coll. «Champs».

Hervieu-Léger D., 2003, Catholicisme, la fin d'un monde, Paris, Bayard.

Huygen J.-M., 2008, « La poubelle et l'architecte », Arles, Actes Sud Beaux Arts, coll. « L'Impensé ».

Kronenburg R., 2007, Flexible, une architecture pour répondre au changement, Paris, Norma Éditions. Lazzarotti O., 2003, « Patrimoine », in Levy J., Lussault M. (dir), Dictionnaire de la géographie et de l'espace des sociétés, Paris, Belin, p. 692-693.

Letourneau J., 2005, « Préface » in Noppen L., Morisset L.K., Les églises du Québec : un patrimoine à réinventer, Sainte-Foy, Québec, Canada, Presses de l'université du Québec.

Maurey H., 2015, Les collectivités territoriales et le financement des lieux de culte, Paris, Sénat.

Ministère de la Culture et de la Communication, 2017, département des études, de la prospective et des statistiques, Chiffres clés statistiques de la culture et de la communication 2017.

Mongeard L., 2015, « La démolition : une pratique urbaine durable ? », Rencontres interdisciplinaires doctorales de l'architecture et de l'aménagement durables, 17 mars 2015, École nationale des travaux publics et École nationale supérieure d'architecture de Lyon, Vaulx-en-Velin.

Namias O., 2016, «Réemploi : Quand l'architecte part à la chasse aux matériaux », Architectures CREE, $\mathrm{n}^{\circ} 376, \mathrm{p} .112-119$.

Noppen L., 2006, « La conversion des églises au Québec. Enjeux et défis », in Coomans T., Morisset L.K., Noppen L., Quel avenir pour quelles églises? What future for which churches?, Québec, Canada, Presses de l'université du Québec, coll. «Patrimoine urbain », p. 275-300.

Noppen L., 2013, « Dossier Explorations autour du destin des églises du Québec », in L'Action nationale, volume CIII, $\mathrm{n}^{\circ}$ 6, p. 54-111.

Noppen L., 2008, « L'église Sainte-Brigide-de-Kildare (Montréal) : étude historique, analyse architecturale et évaluation patrimoniale », chaire de recherche du Canada en patrimoine urbain - ESG-UQAM/Centre communautaire Sainte-Brigide, 236 p.

Perrin A., 2005, L'Église catholique et les églises en régime français de laïcité, thèse de doctorat, sociologie de sciences des religions, École pratique des hautes études $\mathrm{V}^{\mathrm{e}}$ Section, Paris.

Stein V., 2012, » Sauvegarde du patrimoine et développement durable : entre complémentarités et conflits ", in Clavairolle F. et al., Construction politique et sociale des territoires, analyser la patrimonialisation des espaces urbains, $\mathrm{n}^{\circ} 1$, p. 59-69. 
Veschambre V., 2008, Traces et mémoires urbaines : enjeux sociaux de la patrimonialisation et de la démolition, Rennes, Presses universitaires de Rennes, coll. « Géographie sociale ».

\section{NOTES}

1. Sources de l'Institut français d'opinion publique, Analyse : le catholicisme en France en 2010, 2010, p. 4. URL : http://www.ifop.fr/media/pressdocument/238-1-document_file.pdf, (consulté le 05/03/2016).

2. Un ouvrage est publié suite à ce colloque (Coomans T., Morisset L.K. et Noppen L., 2006).

3. Processus amorcé notamment par la Révolution tranquille dès 1960, étape importante dans la société québécoise. En résumé, après l'élection de Jean Lesage, du Parti Libéral Québécois, de grandes réformes sociétales sont entreprises comme la séparation de l'Église catholique et de l'État, l'adoption des principes de l'État providence et la construction d'une nouvelle identité nationale québécoise.

4. Observatoire du patrimoine religieux «Églises, un patrimoine à vendre»: http:// www.patrimoine-religieux.fr/rubriques/gauche/presse/actualites-mediatiques/eglises-unpatrimoine-a-vendre/, (consulté le 15/10/2016).

5. Sondage "Les Français et le patrimoine de proximité », mené pour la Fondation du Patrimoine par la Caisse d'Épargne et Opinion Way en janvier 2016. Question posée aux enquêtés déclarés catholiques: "Certaines églises non entretenues sont réhabilitées en bâtiments civils. Personnellement, y êtes-vous très favorable, assez favorable, peu favorable ou pas du tout favorable?». Au total, $71 \%$ sont dit favorables (16\% «très favorables » et $55 \%$ «assez favorables ») contre $28 \%$ défavorables.

6. «Entre valeur affective et valeur d'usage, quel avenir pour les églises paroissiales ? La région urbaine Lyon Saint-Étienne interrogée par le référentiel du "Plan églises" québécois ", sous la direction de Veschambre V., financée par la Région Auvergne-Rhône-Alpes.

7. Ne sont pas prises en compte les chapelles conventuelles, institutionnelles et cathédrales. La notion de paroisse induit une implantation et un maillage territorial réfléchi. La notion de territoire et de local nous intéresse tout particulièrement dans la diffusion de l'idée d'une reconversion des églises ancrée dans une communauté et les besoins d'un quartier.

8. Rapport issu des travaux de la Commission mondiale pour l'environnement et le développement, intitulé "Our common future», qui consacre et précise le concept de développement durable tel que nous le connaissons aujourd'hui.

9. Ce sont les termes de la loi relative à la solidarité et au renouvellement urbains, SRU (article L121-1 du code de l'urbanisme).

10. Unesco, déclaration de Budapest sur le patrimoine mondial, CONF 202 9, en ligne: http:// whc.unesco.org/fr/decisions/1217/ (consulté le 03/10/2017).

11. La très grande majorité : celles qui appartenaient à l'Église catholique. Par exemple, la basilique de Fourvière, pourtant construite avant 1905, ne fait pas partie du patrimoine de la Ville de Lyon. Elle appartient à une fondation privée.

12. Pour plus de détails sur cette gestion juridique, particulière à la France, voir Meynier-Philip M., 2017, «La conversion architecturale des églises paroissiales à l'épreuve de leurs régimes juridiques", mémoire de diplôme universitaire "Religion, liberté religieuse et laïcité ", université Jean-Moulin Lyon 3 et UCLY, 48 p.

13. Code du droit canonique, canon 1212.

14. Voir Code du patrimoine, Livre VI : Monuments historiques, sites et espaces protégés.

15. Sauf exception, par exemple sur la Région Auvergne-Rhône-Alpes, des églises construites par Le Corbusier ou Maurice Novarina font l'objet de protection au titre des monuments historiques, ou possèdent le label « Architecture contemporaine remarquable ». 
16. Graphique $1:$ « Nombre de mesures de protection de monuments prises, 1990-2015 », ministère de la Culture et de la Communication, département des études, de la prospective et des statistiques, 2017, Chiffres clés Statistiques de la culture et de la communication 2017, p. 147.

17. Il remplace le label « Patrimoine $\mathrm{xx}^{\mathrm{e}} »$ depuis 2016.

18. Article R650-6 du Code du patrimoine. Par exemple: le décret prévoit une obligation d'information du préfet de Région par le propriétaire en cas de travaux sur le bien labellisé, ou en cas de mutation de propriété.

19. Dans le cadre de sa thèse, Anne Perrin a démontré, à travers un sondage effectué dans la rue, que les caractères «roman» et «gothique » reviennent principalement pour qualifier l'église préférée des Français.

20. Date retenue dans le cadre de ce travail, car il s'agit d'une destruction envisagée dans le cadre d'un projet urbain de grande ampleur. L'église Saint-Charles de Serin à Lyon a été détruite et reconstruite à un autre emplacement, pour permettre la création du tunnel de la Croix-Rousse.

21. Direction régionale des affaires culturelles, service de l'inventaire général du patrimoine culturel par exemple.

22. Ces édifices n'ont généralement aucune protection patrimoniale. Le label « Patrimoine $\mathrm{xx}^{\mathrm{e}}$ », devenu en 2016 le label «Architecture contemporaine remarquable » avec la loi relative à la liberté de la création, à l'architecture et au patrimoine, est utilisé comme une reconnaissance mais ne constitue pas une protection juridique.

23. Elle a été radiée uniquement en 2011.

24. Référendum sur la démolition ou restauration de l'église de Saint-Chamond en 2009, démolition contestée de l'église de Gesté en 2013.

25. Meynier-Philip M., 2017, « Communauté patrimoniale et spécialistes, une alliance édifiante? L'exemple d'une communauté pour la défense successive de deux églises à Villeurbanne", colloque Les communautés patrimoniales, Heritage communities, $12^{\mathrm{e}}$ Rencontre internationale des jeunes chercheurs en patrimoine urbain, 28 septembre 2017, chaire de recherche du Canada en patrimoine urbain, université du Québec à Montréal, Montréal, publication à paraître.

26. Après la Seconde Guerre mondiale, la France est en pleine expansion économique, démographique et urbanistique. Le diocèse de Lyon créa en 1957 l'Office diocésain des paroisses nouvelles pour anticiper l'implantation territoriale de futures paroisses voulues au centre des quartiers en construction, près des écoles et des commerces, séparées par un kilomètre de distance au maximum.

27. Meynier-Philip M., 2016, "Églises $x^{\mathrm{e}}$, un manifeste de flexibilité architecturale », colloque L'avenir des églises, 20 octobre 2016, École nationale supérieure d'architecture de Lyon, Vaulx-enVelin.

28. Pétition en ligne: http://www.mesopinions.com/petition/art-culture/demolition-egliseferrandiere-eglise-ur-immacule/12994 (consultée le 25/01/2017).

29. Maîtres d'ouvrage Est Métropole Habitat et Alynéa, maître d'œuvre A-MAS Architecture.

30. Pétition en version papier diffusée dans le quartier (179 signatures) et en ligne (374 signatures) : https://www.mesopinions.com/petition/art-culture/demolition-eglise-ferrandiereeglise-ur-immacule/12994 (consulté le 5 septembre 2017).

31. Église du Cœur Immaculé de Marie (Villeurbanne, la Ferrandière) : ou comment faire rimer destruction avec valorisation du patrimoine, 21 mars 2014 ; Église de la Ferrandière (Cœur Immaculé de Marie à Villeurbanne) Le permis de démolir est affiché..., 22 septembre 2014 ; Église de la Ferrandière : mobilisation - un premier tract, 10 octobre 2014 ; Non à la démolition de l'église de la Ferrandière (Cœur Immaculé de Marie) à Villeurbanne : la pétition est en ligne !, 14 octobre 2014 ; Démolition de l'église de la Ferrandière (Cœur Immaculé de Marie à Villeurbanne) Des nouvelles des pétitions et du tractage, 31 octobre 2014 ; voir en ligne :

http://assocadreviepatrimoine.over-blog.fr/tag/eglises\%20a\%20demolir\%20a\%20villeurbanne/, consulté le 8 septembre 2017. 
32. Conseil de l'Europe, » Convention-cadre du Conseil de l'Europe sur la valeur du patrimoine culturel pour la société, dite "Convention de Faro" ", texte en ligne : https://www.coe.int/fr/ web/conventions/full-list/-/conventions/rms/0900001680083748, consulté le 26 juillet 2017.

33. Historique de l'église du Cœur Immaculé de Marie, article sur l'encyclopédie Le Rize + : http://lerizeplus.villeurbanne.fr/arkotheque/client/am_lerize/encyclopedie/fiche.php?ref=68, (consulté le 25/01/2017).

34. Note de présentation du 34 rue Richelieu, Est Métropole Habitat, pré-étude menée par l'agence d'architecture Patriarche\&Co, 17/01/2012.

35. Entretien avec Stéphanie David, l'architecte chef du projet, le 26/01/2016.

36. Fiche projet site internet agence A-MAS, (consultée le 06/01/2016).

37. Note de présentation du 34 rue Richelieu, Est Métropole Habitat : respecter le référentiel QEB région, suivre la charte fixant les exigences de Qualité environnementale des bâtiments établie par le Grand Lyon.

38. Note méthodologique, équipe A-MAS/GBA/BOST, Saint-Étienne, le 4 /02/2013.

39. Le passé est employé car suite aux contestations du projet par les riverains, le budget a dû s'adapter pour répondre aux demandes des recours du permis de construire. Le projet a dû intégrer la construction de plusieurs murs en limites de parcelle pour couper la vue entre les logements d'accueil et les logements existants. Le budget ainsi alourdi, il ne permettait plus une telle récupération in situ, entretien avec Stéphanie David, architecte du projet, le 27/06/2017.

40. La gestion et la prise en charge de ces gravats forment un sujet à part entière et ne pourront être abordés dans cet article. Cependant, à titre d'information, en 2008, les déchets de démolition représentaient 31,2 millions de tonnes en France, contre 35 millions de tonnes pour les déchets municipaux, voir Mongeard L., 2015, «La démolition: une pratique urbaine durable?», Rencontres interdisciplinaires doctorales de l'architecture et de l'aménagement durables, 17 mars 2015, École nationale des travaux publics et École nationale supérieure d'architecture de Lyon, Vaulxen-Velin.

41. Actuellement, la pratique la plus répandue consiste à enfouir les gravats du gros-œuvre lors de travaux de terrassement pour les autoroutes par exemple.

42. Au niveau international la pratique de l'architecte chinois Wang Shu se démarque. En 2006, lors de la Xe Biennale d'architecture de Venise, il présenta Tiles Garden, une structure de bambou recouverte de tuiles récupérées de bâtiments régionaux détruits. En 2008, il a édifié le musée historique de la ville de Ningbo à partir de briques issues de quartiers traditionnels démolis.

43. La question de l'avenir des objets religieux et du mobilier forme un sujet à part entière. Des pistes sont simplement évoquées ici.

44. C'est le cas pour le diocèse de Saint-Étienne, entretien avec le service immobilier le 24/03/2016.

45. Entretien avec la directrice de la Maison de l'enfance de la Duchère, le 26/09/2016.

46. École d'été du patrimoine, "Les églises comme outils de développement local ", séminaire régional organisé par le Musée régional de Vaudreuil-Soulanges en partenariat avec la chaire de recherche du Canada en patrimoine urbain dans le cadre du «Plan églises » de la vallée du HautSaint-Laurent, le 01/04/2016.

47. La chaire de recherche du Canada en patrimoine urbain de l'université du Québec à Montréal, le Musée régional de Vaudreuil-Soulanges, la Conférence régionale des élus vallée-du-Haut-SaintLaurent (CRÉ), le bureau régional du ministère de la Culture et des Communications et le diocèse de Valleyfield collaborent activement à la recherche historique et l'élaboration de connaissances autour des églises locales.

48. Première évocation dans les médias : «Un "Plan églises" pour le Québec », Le Devoir, 5 août 2014, en ligne : http://www.ledevoir.com/societe/actualites-en-societe/415125/un-plan-eglisespour-le-quebec (consulté le 9 août 2017). 
49. Projet conduit comme service à la collectivité et à ce titre exemplaire et instructif en matière de concertation.

\section{RÉSUMÉS}

Dans un contexte de sécularisation accélérée des sociétés occidentales, des recherches entreprises dans le cadre d'un doctorat en architecture s'interrogent sur l'avenir des églises paroissiales de la région urbaine Lyon Saint-Étienne. Face à une désertion grandissante disparition des paroissiens et de leur usage cultuel -, leur avenir est envisagé grâce à leur valeur d'usage, liée au potentiel de reconversion, comme à leur valeur affective, portée par une communauté patrimoniale. Ce phénomène mérite d'être appréhendé dans une vision globale prenant en compte les problématiques de renouvellement urbain et de densification comme de développement durable. Suite à un inventaire sur le territoire d'investigation, des cas d'étude illustrent les pratiques actuelles : la reconversion et la démolition. Ici, la question des enjeux environnementaux est abordée à travers le renouvellement des édifices qui sont à l'origine du développement et de la mémoire de nos centres urbains et ruraux.

Within the framework of a PhD in Architecture, the research tackles the future of the parochial churches of the Lyon Saint-Étienne urban area, in a context of accelerated secularization of Western societies. Faced with an increasing desertion - disappearance of parishioners and their religious use -their use value, linked to their potential of reconversion, as well as to their affective value, carried by a heritage community, allow them to have a future. We suggest analyzing it in a global vision taking into account the problems of urban renewal, densification and sustainable development. An inventory was made of the Lyon Saint-Étienne urban area, and those case studies illustrate current practices : reconversion and demolition. Here, this research addresses the environmental issues through the renewal of the buildings that are at the origin of the development and of the memory of the urban cores and the villages.

\section{INDEX}

Mots-clés : patrimoine, église paroissiale, démolition, reconversion, réemploi, déchet, patrimonialisation, développement durable, développement local, communauté patrimoniale Keywords : heritage, paroquial church, demolition, reconversion, re-use, waste, patrimonialization, sustainable development, local development, heritage community

\section{AUTEUR}

\section{MÉLANIE MEYNIER-PHILIP}

Mélanie Meynier-Philip est architecte diplômée d'État HMONP (Habilitation à exercer la maîtrise d'œuvre en son nom propre), doctorante en architecture, elle s'intéresse à la patrimonialisation par la reconversion. Après une expérience à la chaire de recherche du Canada en patrimoine urbain, sa thèse porte sur l'avenir des églises autour de Lyon Saint-Étienne. Elle appartient au 
laboratoire EVS-LAURE UMR 5600 (Environnement Ville et Société - Lyon Architecture Urbanisme REcherche)/École nationale supérieure d'architecture de Lyon.

melanie.meynier@lyon.archi.fr 